\title{
Analysis of high-throughput screening reveals the effect of surface topographies on cellular morphology
}

Citation for published version (APA):

Hulsman, M., Hulshof, F., Unadkat, H., Papenburg, B. J., Stamatialis, D. F., Truckenmuller, R., van

Blitterswijk, C., de Boer, J., \& Reinders, M. J. T. (2015). Analysis of high-throughput screening reveals the effect of surface topographies on cellular morphology. Acta Biomaterialia, 15, 29-38.

https://doi.org/10.1016/j.actbio.2014.12.019

Document status and date:

Published: 15/03/2015

DOI:

10.1016/j.actbio.2014.12.019

Document Version:

Publisher's PDF, also known as Version of record

Document license:

Taverne

Please check the document version of this publication:

- A submitted manuscript is the version of the article upon submission and before peer-review. There can be important differences between the submitted version and the official published version of record.

People interested in the research are advised to contact the author for the final version of the publication, or visit the DOI to the publisher's website.

- The final author version and the galley proof are versions of the publication after peer review.

- The final published version features the final layout of the paper including the volume, issue and page numbers.

Link to publication

\footnotetext{
General rights rights.

- You may freely distribute the URL identifying the publication in the public portal. please follow below link for the End User Agreement:

www.umlib.nl/taverne-license

Take down policy

If you believe that this document breaches copyright please contact us at:

repository@maastrichtuniversity.nl

providing details and we will investigate your claim.
}

Copyright and moral rights for the publications made accessible in the public portal are retained by the authors and/or other copyright owners and it is a condition of accessing publications that users recognise and abide by the legal requirements associated with these

- Users may download and print one copy of any publication from the public portal for the purpose of private study or research.

- You may not further distribute the material or use it for any profit-making activity or commercial gain

If the publication is distributed under the terms of Article $25 \mathrm{fa}$ of the Dutch Copyright Act, indicated by the "Taverne" license above, 


\title{
Analysis of high-throughput screening reveals the effect of surface topographies on cellular morphology
}

\author{
Marc Hulsman $^{\mathrm{a}, *}$, Frits Hulshof ${ }^{\mathrm{b}, \mathrm{c}}$, Hemant Unadkat ${ }^{\mathrm{b}, 1}$, Bernke J. Papenburg ${ }^{\mathrm{e}}$, Dimitrios F. Stamatialis ${ }^{\mathrm{c}}$, \\ Roman Truckenmüller ${ }^{\mathrm{b}}$, Clemens van Blitterswijk ${ }^{\mathrm{b}}$, Jan de Boer ${ }^{\mathrm{b}, \mathrm{d}}$, Marcel J.T. Reinders ${ }^{\mathrm{a}}$ \\ a Delft Bioinformatics Lab, Delft University of Technology, Mekelweg 4, Delft 2628 CD, The Netherlands \\ ${ }^{\mathrm{b}}$ MIRA Institute for Biomedical Technology and Technical Medicine, Department of Tissue Regeneration, University of Twente, P.O. Box 217, Enschede 7500 AE, The Netherlands \\ ${ }^{\mathrm{C}}$ MIRA Institute for Biomedical Technology and Technical Medicine, Department of Biomaterials Science and Technology, University of Twente, P.O. Box 217, Enschede 7500 AE, \\ The Netherlands \\ ${ }^{\mathrm{d}}$ Laboratory for Cell Biology-inspired Tissue Engineering, Merln Institute, Maastricht University, Minderbroedersberg 4-6, Maastricht 6211 LK, The Netherlands \\ ${ }^{\mathrm{e}}$ Materiomics BV, Professor Bronkhorstlaan 10 - gebouw 48, 3723MB Bilthoven, The Netherlands
}

\section{A R T I C L E I N F O}

Article history:

Received 24 September 2014

Received in revised form 5 December 2014

Accepted 19 December 2014

Available online 30 December 2014

\section{Keywords:}

Cell morphology

Interface

Surface topography

Modelling

Mesenchymal stem cell

\begin{abstract}
A B S T R A C T
Surface topographies of materials considerably impact cellular behavior as they have been shown to affect cell growth, provide cell guidance, and even induce cell differentiation. Consequently, for successful application in tissue engineering, the contact interface of biomaterials needs to be optimized to induce the required cell behavior. However, a rational design of biomaterial surfaces is severely hampered because knowledge is lacking on the underlying biological mechanisms. Therefore, we previously developed a high-throughput screening device (TopoChip) that measures cell responses to large libraries of parameterized topographical material surfaces. Here, we introduce a computational analysis of highthroughput materiome data to capture the relationship between the surface topographies of materials and cellular morphology. We apply robust statistical techniques to find surface topographies that best promote a certain specified cellular response. By augmenting surface screening with data-driven modeling, we determine which properties of the surface topographies influence the morphological properties of the cells. With this information, we build models that predict the cellular response to surface topographies that have not yet been measured. We analyze cellular morphology on 2176 surfaces, and find that the surface topography significantly affects various cellular properties, including the roundness and size of the nucleus, as well as the perimeter and orientation of the cells. Our learned models capture and accurately predict these relationships and reveal a spectrum of topographies that induce various levels of cellular morphologies. Taken together, this novel approach of high-throughput screening of materials and subsequent analysis opens up possibilities for a rational design of biomaterial surfaces.
\end{abstract}

(c) 2015 Acta Materialia Inc. Published by Elsevier Ltd. All rights reserved.

\section{Introduction}

High-throughput screening has become a valuable tool for biologists. Examples of such tools range from drug discovery platforms [1] to microfluidic cell-based assays [2]. In situations where biological mechanisms are unknown and/or hard to simulate, such screenings can help identify promising directions for new research.

Recently, we reported on the development of such a screening platform for biomaterial surface topographies [3]. Biomaterials play an important role in tissue engineering, as their surface has been

\footnotetext{
* Corresponding author. Tel.: +31 152782522.

E-mail address: m.hulsman@tudelft.nl (M. Hulsman).

1 Present address: Mechanobiology Institute, National University of Singapore, 5A Engineering Drive 1, Singapore 117411, Singapore.
}

shown to affect cell behavior [4-7]. Such behavior can, for example, lead to non-optimal functioning of implants (e.g. by encapsulating an orthopedic implant with fibrous tissue). By control of cell morphology through surface-based adhesion contrasts, it has moreover been shown that surfaces can affect cell proliferation and even cell differentiation. For example, the available space for spreading [8] and the shape of this space $[7,9]$ can affect the differentiation of respectively human and rat mesenchymal stem cells (hMSCs/ rMSCs), steering them towards either osteogenesis or adipogenesis.

Cell morphologies can also be modulated through modification of the surface topography. Topographies have been shown to affect cell attachment (e.g. [10,11]), cell viability (e.g. [12]) and also cell differentiation (e.g. [13-15]). Another example of the use of surface topographies was given in Ref. [16], where microgroove surface topographies were used to align cells, allowing them to form a 
healthy collagen matrix. This is relevant to the healing of ligament and tendons, in which unaligned cells negatively affect the structure of the collagen matrix. Similarly, in Ref. [17], the focus was on replacement materials for bladder tissue. It was shown that the use of a nanostructured material enhanced cell adhesion and cell growth as well as elastin and collagen production. Surface topographies are thus important tools in the engineering of replacement tissues, such as muscles, bone and blood vesicles.

Finding surfaces that instruct cells to show a certain behavior is, however, a challenging task, as the number of ways in which materials can be modified is virtually unlimited and the biological mechanisms behind cell-material interactions are not yet fully understood. This implies that there is a need for a screening approach in which one can measure the reactions of living cells to a large set of material surfaces. The main focus to date has been on the creation of surface topography gradients (e.g. $[11,13,14]$ ). These gradients allow one to explore cellular responses across the full range of a certain fabrication parameter within a single experiment. More complex gradients can also be constructed, such as the 2-D gradient explored in Ref. [18]. However, the range of possible surface topographies is large, and many design parameters and their interactions can be considered. Such high-dimensional parameter spaces become difficult to explore using gradients. Combinatorial (high-throughput) screenings [19] have been identified as a possible solution to these problems, and have successfully been applied for polymer-based biomaterial development [20].

In this regard, we have developed a chip (called TopoChip) on which cells are grown within units (TopoUnits), each containing a different patterned surface topography. In total, the chip contains 2176 distinct surfaces, each measured by two TopoUnits. Highthroughput fluorescence microscopy is used to determine cellular responses at the single-cell level.

With the miniaturization (realized through the use of a chipbased platform), combined with an algorithmic design of surface topographies, the effects of numerous surface design parameters can be explored within a single experiment. Through the standardization of chip formats, the results of different experiments can be compared.

Such a new high-throughput platform also reveals opportunities for new computational analysis methods. The large amount of information produced enables a transition whereby surface screening is augmented by surface modeling. That is, we can begin to link the biological performance of a surface to its actual topographic design. This systematic approach to material research, in which one combines screening with modeling, has been termed "materiomics" [21]. This gives insights that open the road to a rational approach to surface discovery, which is useful, given that the potential materiome is of infinite size.

In this work, we propose a materiomics-based computational analysis for the TopoChip. To illustrate our analysis methods, we explore the cell morphological responses of hMSCs (which are known to be mechanosensitive) to different surface designs. We learn models which relate such surface designs to the measured cell morphology. In this way, we gain insight into the important surface design properties. This finally allows us to predict cellular responses to surfaces in silico.

We focus specifically on finding "hit" surfaces, i.e. those surfaces that best promote a certain specified cell behavior, as this is one of the primary applications of biomaterial testing. This is a challenging problem in a high-throughput setting, not only due to the number of material surfaces tested, but also due to the miniaturization of the surface area required for designing an efficient high-throughput platform. This means that the number of cells tested per surface type is reduced, making results more susceptible to (natural) variation. Nevertheless, across the whole chip, a large amount of data is gathered for a large number of cells. We propose a hit ranking method that makes use of this data by taking into account surface similarities and cell behavior similarities. This allows us to share information between surfaces, thereby significantly improving hit surface ranking performance and reducing the number of replicate measurements required.

We make use of machine-learning techniques to learn models that link cell responses to surface descriptions. We show how the use of robust statistics enables us to obtain high-quality hit rankings and create predictive models that are consistent across experiments. Over the years, various studies within the (molecular) biomaterials field have investigated the use of machine-learning algorithms for various applications [22], ranging from protein adsorption performance predictions [23] to drug-release kinetics predictions [24]. Their correctness and predictive quality generally depend on the number of measurements that are used to learn the model. With the TopoChip, we now have, for the first time, access to measurements across thousands of surfaces, enabling these methods to learn unprecedentedly detailed models of the cell-surface relationship.

To show how the surfaces affect the morphologies of hMSC cells, we perform in this study an eight-chip experiment, constituting $\sim 35,000$ surfaces, on which half a million hMSCs are grown. By measuring the morphology of each of these cells, we show that the surfaces consistently induce a whole range of different morphologies. We generalize across all measurements to determine how the surfaces influence these morphological responses. Based on this, we generate spectra of surfaces that induce the whole range of possible morphological property values, and show how models allow us to predict these morphological property values for new surfaces in silico.

\section{Material and methods}

\subsection{General approach}

\subsubsection{TopoChip}

The TopoChip, which has dimensions of $2 \times 2 \mathrm{~cm}$, measures the effect of 2176 different topographical surfaces on cell behavior

Table 1

Surface properties.

\begin{tabular}{|c|c|}
\hline $\begin{array}{l}\text { Surface } \\
\text { property }\end{array}$ & Description \\
\hline FeatSize & $\begin{array}{l}\text { The size of the bounding square for the primitives }(10,20 \text { or } \\
28 \mu \mathrm{m})\end{array}$ \\
\hline NumCirc & The number of circles used \\
\hline NumTri & The number of triangles used \\
\hline NumLine & The number of lines used \\
\hline CircDiam & Circle diameter \\
\hline TriSize & Length of the shortest side of a triangle \\
\hline LineLen & Line length \\
\hline RotSD & $\begin{array}{l}\text { The standard deviation (in degrees), is used to determine the } \\
\text { rotation of the primitives when they are placed in the feature }\end{array}$ \\
\hline Rot & $\begin{array}{l}\text { The standard deviation for rotation of primitives scaled with } \\
\text { number of line and triangle primitives (since circle primitives } \\
\text { are unaffected by rotation) }\end{array}$ \\
\hline $\begin{array}{r}\text { WN0.1- } \\
\text { WN4 }\end{array}$ & $\begin{array}{l}\text { The fraction of energy in the signal in sinusoids with } \\
\text { wavenumber } 0.1-4\end{array}$ \\
\hline CircArea & The area of circle primitives \\
\hline TriArea & The area of triangle primitives \\
\hline LineArea & The area of line primitives \\
\hline DC & The number of circle primitives scaled by feature area \\
\hline DT & The number of triangle primitives scaled by feature area \\
\hline DL & The number of line primitives scaled by feature area \\
\hline $\mathrm{CA}$ & The total area of circle primitives scaled by feature area \\
\hline $\mathrm{TA}$ & The total area of triangle primitives scaled by feature area \\
\hline LA & The total area of line primitives scaled by feature area \\
\hline CCD & Number of color changes of the feature over the diagonal \\
\hline
\end{tabular}

Feature area refers to the feature size, which is a bounding square of 10,20 or $28 \mu \mathrm{m}$ (see FeatSize). Each feature contains primitives (circles, triangles and lines (rectangles)). Features are repeated to cover the surface of a TopoUnit. 


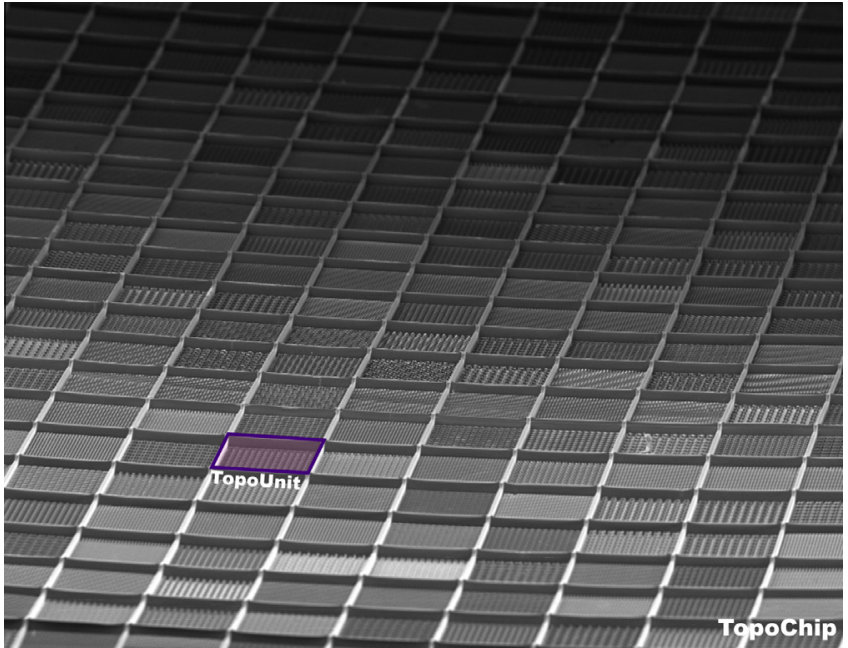

Fig. 1. The TopoChip. EM image of the TopoChip, showing the different surface topographies in each unit. Each chip consists of 4356 TopoUnits (2176 unique topographies in duplicate + four blanks).

(Fig. 1). Each surface consists of a specific feature, which is repeated in both the horizontal and vertical directions to create a surface pattern. A feature consists of a variable number of primitives: circles, triangles and rectangles. Each of these surfaces is placed twice on the chip, in rectangular bordered areas called TopoUnits. Surfaces are constructed algorithmically, and characterized through different properties (Table 1 ). TopoChips are made by hot embossing a $250 \mu \mathrm{m}$ thick (poly)lactic acid film (Folienwerk Wolfen $\mathrm{GmbH}$ ) with a silicon mould containing the inverse pattern. The silicon mould is fabricated by photolithography and deep reactive etching, as described in Ref. [3]. Cells are cultured on the chip and imaged using fluorescence microscopy.

\subsubsection{Data acquisition}

To determine the response of the cells to each of the surfaces on the chip, we quantify the cells in each TopoUnit at the single-cell level. First, raw chip images are pre-processed to correct for imaging artifacts and quantify quality issues (see Appendix B), after which images of the individual TopoUnits on the chip are created. Next, we used Cell Profiler to segment (see Section 2.2 in Appendix B) and characterize cells at the single-cell level, using various descriptors describing both the cell and the nucleus shape (Table 2). As a result, we obtain cell responses for all distinct surfaces (16 replicates each), with all surface replicates (TopoUnits) being described through the following measures: (i) cell counts and 32 morphological properties for all the cells (Table 2); (ii) 30 properties describing various aspects of the TopoUnit surface (Table 1); and (iii) a set of quality control properties (see Table B.1 in Appen$\operatorname{dix} B)$.

\subsubsection{Data analysis}

Then surface-cell response models are learned based on the topographic properties and cell/nucleus descriptors available for each TopoUnit. We rely on robust statistical analysis and machine-learning techniques to be able to deal with the noisy data as a result of the low number of cells in a TopoUnit and to derive predictive models for the cell-surface associations.

\subsection{Data acquisition}

\subsubsection{TopoChip cell seeding and staining}

Eight TopoChips were seeded with passage 3 hMSCs (Lonza) at a density of 4000 cells $\mathrm{cm}^{-2}$. The TopoChip experiment as well as
Table 2

Morphological measures.

\begin{tabular}{|c|c|}
\hline $\begin{array}{l}\text { Cell morphology } \\
\text { property }\end{array}$ & Description \\
\hline Area & Number of pixels in a region \\
\hline Eccentricity & $\begin{array}{l}\text { The eccentricity of the ellipse that has the same second- } \\
\text { moments as the region. Eccentricity is the ratio of the } \\
\text { distance between the foci of the ellipse and its major } \\
\text { axis length }\end{array}$ \\
\hline EulerNumber & $\begin{array}{l}\text { The number of separate components in the region } \\
\text { minus the number of holes in those components }\end{array}$ \\
\hline Extent & $\begin{array}{l}\text { The proportion of pixels in the bounding box that are } \\
\text { also in the region }\end{array}$ \\
\hline FormFactor & $\begin{array}{l}\text { Calculated as } 4 \pi \times \text { area/perimeter }{ }^{2} \text {. Equals } 1 \text { for a } \\
\text { circular object }\end{array}$ \\
\hline IntegratedIntensity & The sum of the pixel intensities within the region \\
\hline MajorAxisLength & $\begin{array}{l}\text { Length (in pixels) of the major axis of the ellipse that } \\
\text { has the same normalized second central moments of } \\
\text { the region }\end{array}$ \\
\hline MassDisplacement & $\begin{array}{l}\text { The distance between the centers of gravity in the gray- } \\
\text { level representation of the object and the binary } \\
\text { representation of the object }\end{array}$ \\
\hline MaxIntensity & Maximal intensity of the pixels within the region \\
\hline MinorAxisLength & $\begin{array}{l}\text { Length (in pixels) of the minor axis of the ellipse that } \\
\text { has the same normalized second central moments of } \\
\text { the region }\end{array}$ \\
\hline Orientation & $\begin{array}{l}\text { The angle (from }-90 \text { to } 90^{\circ} \text { ) between the } x \text {-axis and the } \\
\text { major axis of the ellipse that has the same second } \\
\text { moments as the region }\end{array}$ \\
\hline Perimeter & $\begin{array}{l}\text { The total number of pixels around the boundary of a } \\
\text { region }\end{array}$ \\
\hline Solidity & $\begin{array}{l}\text { The proportion of pixels in the convex hull that are also } \\
\text { in the region }\end{array}$ \\
\hline
\end{tabular}

The morphological measures, determined using Cell Profiler [14], are used to represent the nuclei shapes (DNA staining) and cell shapes (actin staining). Full measures are described as "Region Measure StainingLocation", with Region being Cell or Nucleus, Measure being a measure from this table, and StainingLocation being Nucleus or Cytoplasm. For example, Nucleus MaxIntensity Cytoplasm describes, within the nucleus region, the maximum intensity of the actin staining within the cytoplasm.

previous cell expansions were performed in $\alpha$-minimal essential medium (Gibco, 22-571-038) supplemented with $10 \%$ fetal bovine serum (Lonza), $2 \mathrm{mM}$ L-glutamine (Gibco, 25030), $0.2 \mathrm{mM}$ ascorbic acid (Sigma, A8960) and $100 \mathrm{U} \mathrm{ml}^{-1}$ penicillin $+100 \mathrm{~g} \mathrm{ml}^{-1}$ streptomycin (Gibco, 15140-122). After a growth period of 7 days, the cells were fixed with $10 \%$ formalin for $15 \mathrm{~min}$. After fixation the cells were permeabilized with $0.1 \%$ Triton X-100 for $10 \mathrm{~min}$, blocked with $4 \%$ bovine albumin serum and incubated with phalloidin-Alexa488 (Invitrogen) 1:100 for $30 \mathrm{~min}$ and 4',6-diamidino-2-phenylindole (DAPI; Thermo Fisher) for $10 \mathrm{~min}$, before being mounted in Mowiol mounting medium (Sigma).

\subsubsection{Imaging}

The chips were imaged using a BD Pathway 435 automated microscope. A total of 4356 images (one for each TopoUnit) were acquired for each chip and fluorescence channel. These were all combined into a large montage (of $62,964 \times 62,964$ pixels, i.e. 〜3964 megapixels).

\subsubsection{Image processing}

Flat-field correction and image normalization (contrast stretching) were performed, and the background was estimated and subtracted. A set of quality control features were also calculated, to be used to detect outliers (see Appendix B). For each TopoUnit and fluorescence image, montage images were cropped into separate images of $800 \times 800$ pixels.

\subsubsection{Measuring cell morphologies}

For each of the TopoUnit images, Cell Profiler [25] was employed to capture the cell morphology of the cells growing in 
that unit. To this end, the DAPI staining was used to recognize nuclei, using the Otsu Adaptive thresholding method. Subsequently, each of these nuclei was used to initiate the search for a corresponding cell, by making use of the Alexa488 staining, Otsu adaptive thresholding and the provided propagation algorithm. Measurements were performed on the resulting shapes. For the final morphology measurements (except for cell alignment), we took the median across all cells in a TopoUnit. Cell alignment per TopoUnit was calculated by aligning all of the cells maximally by turning their orientation 180 degrees, and subsequently calculating the mean absolute deviation over these orientations.

\section{Calculation}

One of the primary uses of high-throughput biomaterial testing is to find "hits", i.e. those material surfaces that best evoke a certain cell response. While a high-throughput chip such as the TopoChip allows one to test many surfaces in parallel, the tested area for each surface is reduced. Consequently, the measured cell morphology is more variable, which can make it difficult to reliably identify hits. Similar to what is commonplace in gene expression microarray testing, however, one can make use of multiple replicates. This allows for an improved estimate of the mean surface performance and also makes it possible to determine the variability of the measurements, which one can use to check the significance of the result and control for outliers.

\subsection{Ranking surface-induced cell responses with a robust statistical test}

The commonly used post hoc analysis of variance (ANOVA) tests only rank surfaces based on their mean performance. We believed that a surface ranking which also takes into account the variance would give improved results, by being more robust to outliers (Section 2.4 of Appendix B describes how this is accomplished). As statistical functions, the Mann-Whitney $U$-test and Welch's $t$-test (allowing unequal variances) are compared to rankings based on the mean (i.e. the same ordering as ANOVA) and the median.

In Fig. 2a, we illustrate the performance of these statistics by ranking surfaces on their effect on the roundness of the nucleus (known as nucleus form factor), as we know that this specific characteristic is influenced by surface topography (Fig. 4). As we do not know the "true ranking", we instead determine, for each statistic, the consistency between two surface rankings. Specifically, we looked at a ranking based on one, two, four or eight replicates, and compared it to a ranking (obtained using the same statistic) that was calculated using eight other replicates (note that there are 16 replicates). As both surface rankings should reflect the same "true ranking", we expect that more consistent rankings reflect a better performing statistic. To capture this, we calculated the Spearman correlation (rank correlation) between these two rankings (averaged over 25 repeats of dividing the 16 replicates into two sets).

We find that robust statistics, based on ranks (both the median and the Mann-Whitney $U$-test), perform significantly better, likely due to their capacity to handle outliers. Furthermore, taking the distribution of the replicate measurements (in the Mann-Whitney $U$-test) into account further improves performance. We see the same results not only for different numbers of chips, but also for other morphological properties (with a mean rank correlation of 0.39 for the Mann-Whitney $U$-test; the next best statistic is the median statistic, with a mean rank correlation of 0.35 ), based on which we use the Mann-Whitney $U$-test as the default ranking method.

\subsection{Model-based ranking of surfaces inducing a specific cellular response}

As indicated in Fig. 2a, a significant number of chips might be required to obtain a good ranking performance. We propose an alternative solution: one can (virtually) increase the number of replicate measurements by sharing information across similar surfaces. This means that we need to determine which surfaces are similar in terms of their influence on a certain cell morphological parameter. We realize this by regressing the measured cell morphology to surface properties using ordinary linear regression, Lasso regression [26], M-estimation regression [27], support vector regression [28] or regression trees (see Section 2.5 of Appendix B for details). Surfaces can then be ordered with the regression model.

Fig. 2b shows the results (evaluated using 10 -fold cross-validation) for the ordinary linear regression, Lasso regression and $\mathrm{M}$ estimation regression models. Note that the ranking consistency of these model-based methods is far higher than those obtained
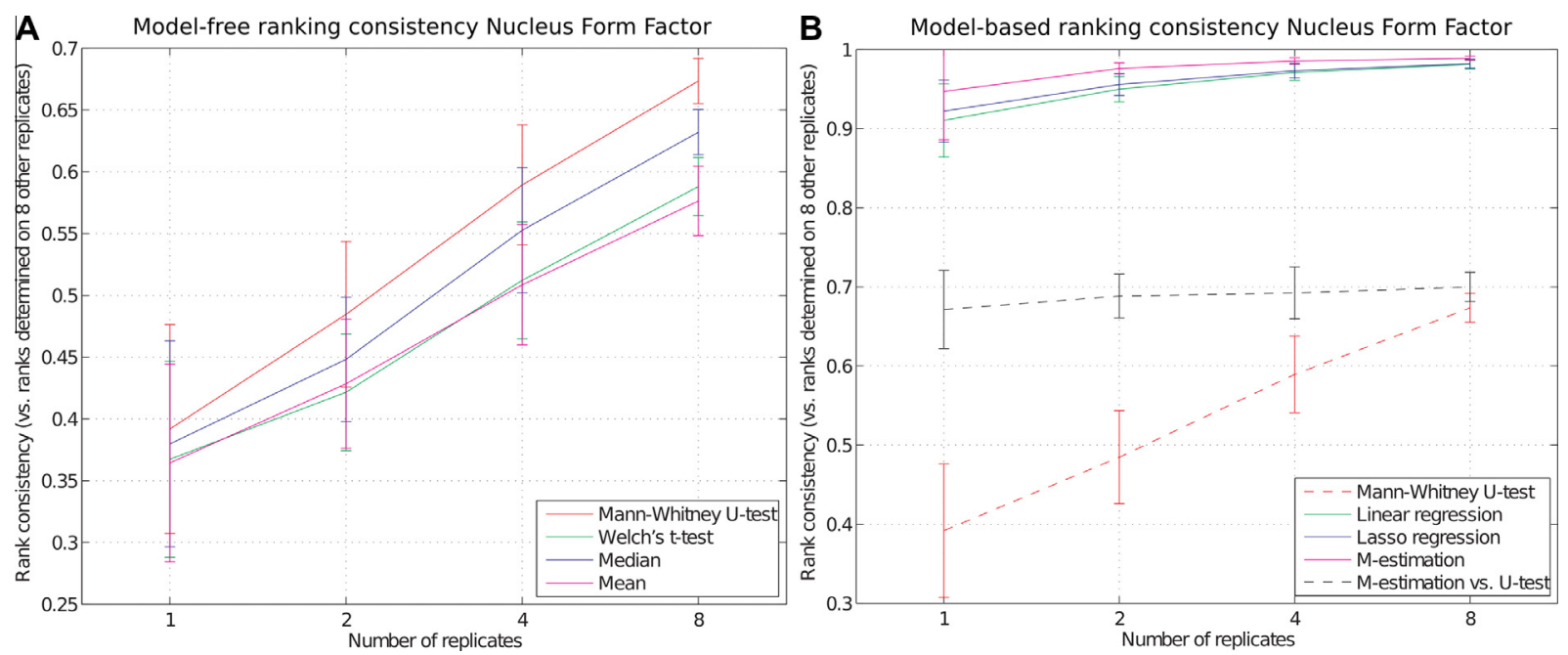

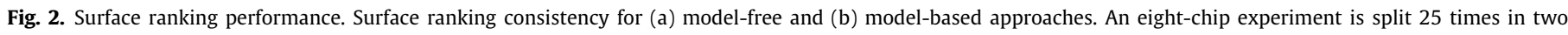

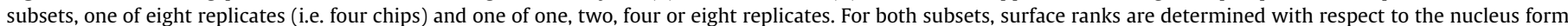

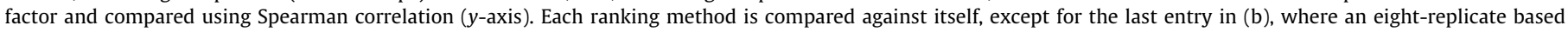
Mann-Whitney $U$-test ranking is compared to an M-estimation regression ranking. 


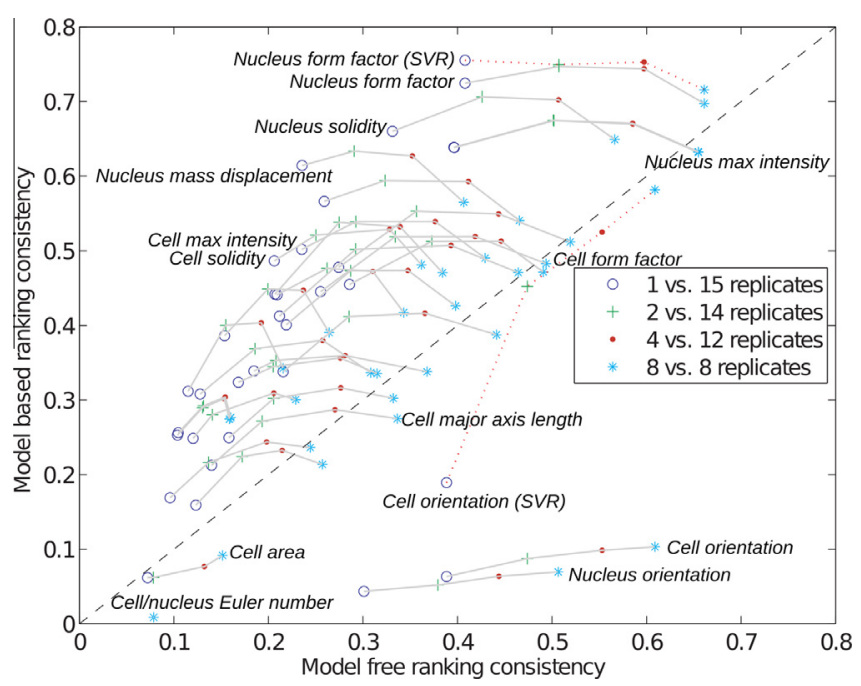

Fig. 3. Model-free vs. model-based ranking consistency, comparing the performance of a model-based (M-estimation regression) ranking with a Mann-Whitney $U$-test ranking and comparing the consistency of both (rank correlation) to a MannWhitney $U$-test ranking on a different set of replicates (replicates selection and ranking repeated 25 times). Each gray line connects the performances of a single morphological parameter. The red dotted lines show performances of the SVR-RBFbased test for Nucleus Form Factor and Cell Orientation. The set of tested morphological properties is the same as in Fig. 4.

using the model-free statistics discussed earlier. Similar to the model-free statistics, we find that the method robust to outliers (M-estimation regression) results in the best consistency, attaining a Spearman correlation of 0.99 . Moreover, the M-estimation regression ranking obtained using two replicates performs better than the Mann-Whitney $U$-test ranking obtained using eight replicates (determined by correlating both to a separate Mann-Whitney $U$-test ranking), indicating that the model-based method has superior ranking performance.
While the model-based approach (M-estimation regression) can give us a much-improved surface ranking, it differs from the model-free approach (Mann-Whitney $U$-test) in that a model of the surface-cell response relations is required. We therefore determined whether the various surface-response relationships can actually be captured in an automatically learned model. To this end, we plotted the performances of the model-based (M-estimation regression) and the model-free (Mann-Whitney $U$-test) ranking against each other (Fig. 3). It shows that, for most morphological properties, the model-based approach performs better or similar compared to the model-free approach, suggesting a correctly learned model. As expected, with increasing numbers of replicates, the model-free approach catches up to the model-based approach. Thus, when enough replicate measurements are available on the surface itself, measurements on similar surfaces (which are used by model-based tests) no longer provide additional information. However, in many practical cases, a large number of replicates will not be available, in which cases the model-based approach is the preferred way to rank surfaces.

For two properties, cell and nucleus orientation, a model-free ranking approach clearly performs better. This is likely due to the fact that surface properties do not include an adequate description of the overall surface primitive orientations. As an alternative solution, we tested a more advanced regression method (support vector regression using a radial basis function kernel, SVR-RBF). The SVR-RBF improves the ranking consistency with the Mann-Whitney $U$-test to 0.59 , as compared to 0.10 for the M-estimation regression (see the red-dotted line in Fig. 3). The support vector regression uses a robust loss function, similar to M-estimation regression, but additionally is able to derive relations based on nonlinear combinations of surface properties, which apparently is necessary to predict cell orientation accurately. The SVR-RBF method, however, has a large computational cost due to the required optimization of three hyper-parameters. Furthermore, it is difficult to interpret the predictive model due to its non-linearity. For these reasons, we use the M-estimation regression in our analyses and use the SVR-RBF only for detailed investigations.

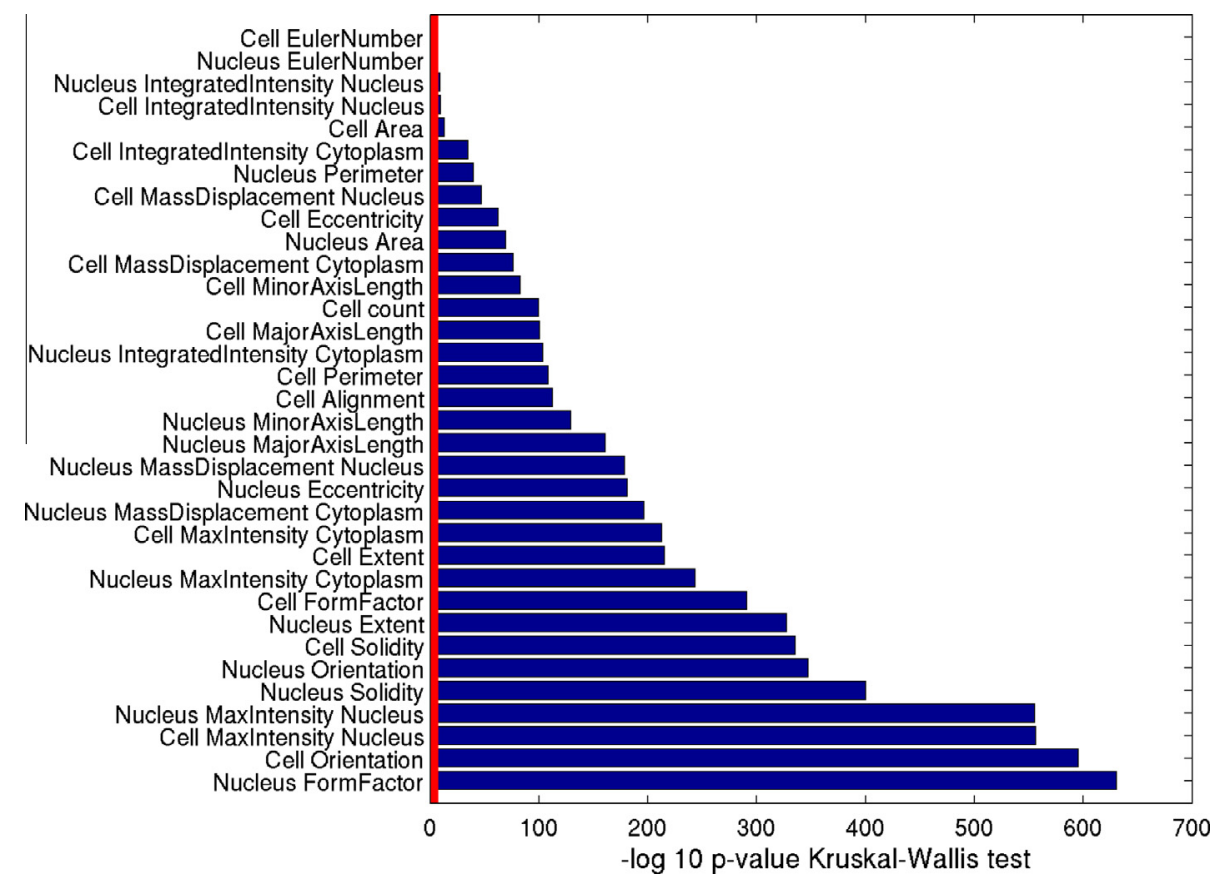

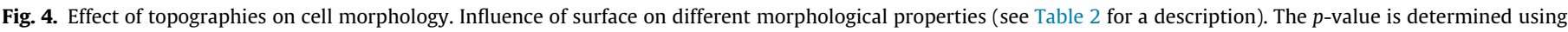
the Kruskal-Wallis test. The red line indicates the 0.05 significance threshold after Bonferoni multiple testing correction $\left(\operatorname{corresponding}^{-} \log _{10} p\right.$-value $\left.=2.8\right)$. 


\section{Results}

\subsection{Surfaces influence cell morphology}

To ascertain whether surfaces actually influence the observed cell responses, we performed a Kruskal-Wallis test [29] for each cell morphological parameter separately against all surface topographies grouped by the replicates. This determines whether a cell morphological parameter differs significantly between the 2176 surfaces as compared to the variations within the replicates.

Fig. 4 shows the results for the 33 morphological properties (Table 2). Even after Bonferroni multiple testing correction [30], the Kruskal-Wallis $p$-values are strongly significant, indicating that most morphological properties are influenced by the surface topography at least to some extent.

From the 33 morphological properties, the nucleus form factor (i.e. the "roundness" of the nucleus, defined as $4 \pi \times$ area/ (perimeter $)^{2}$, where the area and perimeter are measured over the detected nucleus) turns out to have the most significant response to changes in the surface topography (also clearly visible in Fig. 5). The cell orientation and the maximum intensity of the DAPI staining within the cell/nucleus also show highly significant effects.

This can be understood for some morphological properties that do not show a significant effect on changes in surface topographies. "Euler Number", for example, represents the number of holes in the binarized staining image of a cell/nuclei. This has no immediate biological interpretation in the current context, and as such functions as an appropriate negative control. The total amount of DNA staining ("Integrated Intensity Nucleus") is also relatively unaffected by differences in the surfaces, consistent with the expectation that the amount of DNA per cell is unlikely to be influenced by surface topography. On the other hand, "Cell Area" only moderately responds to the surface changes. We should, however, take care in accepting this conclusion, as both technical (e.g. segmentation errors) and natural variation might overwhelm the signal. In particular, the lower significance can be explained to some extent by variations in the cell density per surface (due either to cell attachment or cell proliferation differences). An increase in cell density leads to a decrease in the average area per cell, due to cell overlap and/or competition for space. Correcting for this effect (Section 2.3 in Appendix B) gives rise to a (limited) increase in significance. In contrast, a decrease in significance after this correction is observed for the mass displacement parameters and the major axis length of cells, possibly because these factors are closely correlated to surface designs that have a positive influence on cell density. For other cases, however, the variability in cell density has negligible effects on the results.

\subsection{Ranking surfaces on their effect on cell morphology}

\subsubsection{Non-roundness of nucleus induced by confined, elongated topographic spaces}

We explored which surfaces affect the nucleus roundness (form factor) using the developed model-based SVR-RBF ranking method (see Section 3.3). The topography of the lowest ranked surfaces (left in Fig. 5) suggests that non-round nuclei are induced by confined, elongated, topographical spaces. Using the whole set of surfaces, a rank correlation of 0.41 is obtained between the nucleus form factor and the cell form factor, indicating that deformation of the cell cytoskeleton coincides with nucleus shape deformation. Moreover, nucleus orientation has a correlation coefficient of 0.75 with cell orientation, showing that the elongated nuclei point in the same direction as the elongated cells. This is also clearly noticeable in the fluorescence images of the top three high and low ranking surfaces in Fig. 5. Round nuclei are only obtained when cells have enough space to spread out. Curiously, the two empty surfaces on the chip are not among the top ranked (although they are ranked high at places 50 and 279 using the Mann-Whitney U-test). However, we cannot conclude that the placement of a few primitives on a surface induces extra roundness, as there is a non-significant difference between the response of the top ranked surfaces and the empty surfaces.

Nucleus morphology and cell morphology lose their correlation when the nucleus is directly compacted by the surface structures. We consider the nucleus maximum intensity to be a measure that
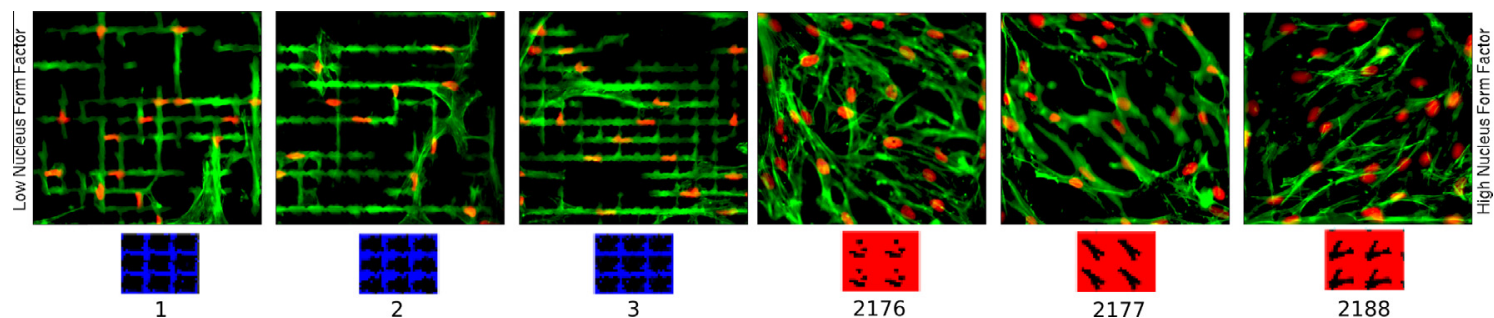

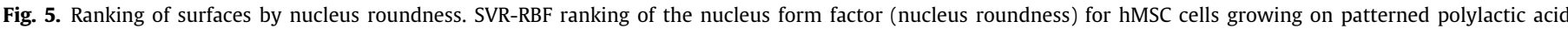

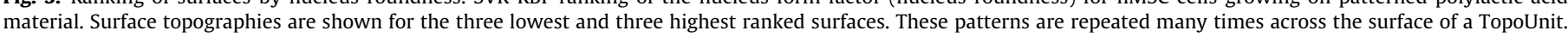

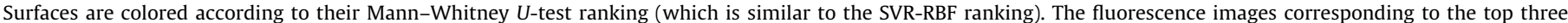
highest and lowest ranked surfaces show the nucleus and cytoskeleton staining in red and green, respectively.
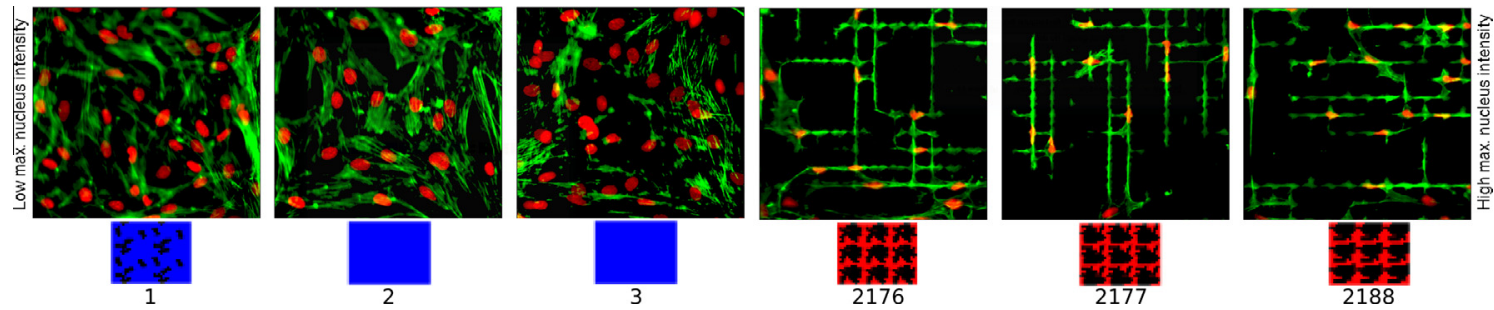

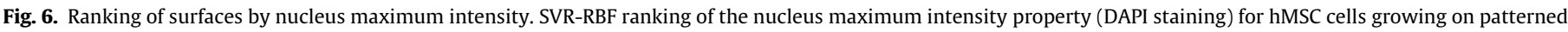
polylactic acid material. See Fig. 5 for more details. 

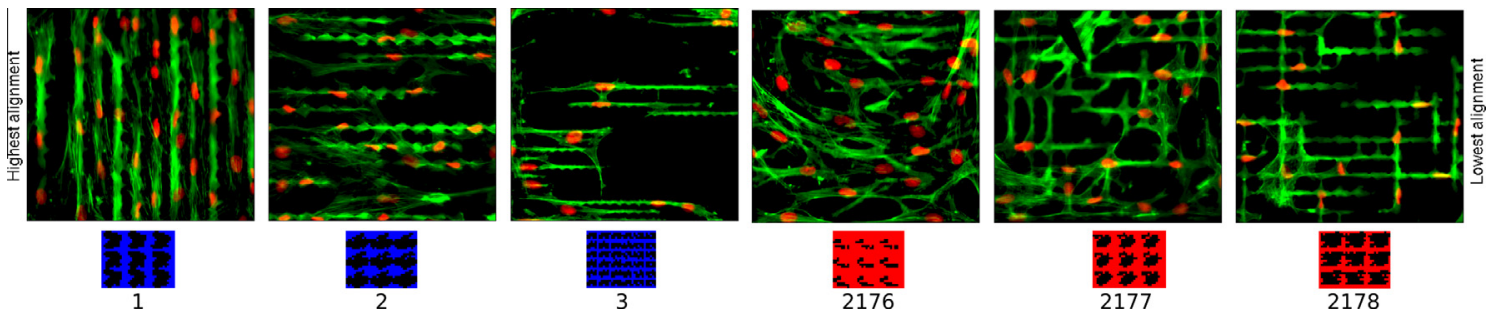

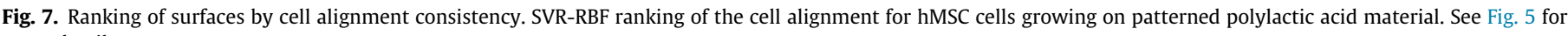
more details.
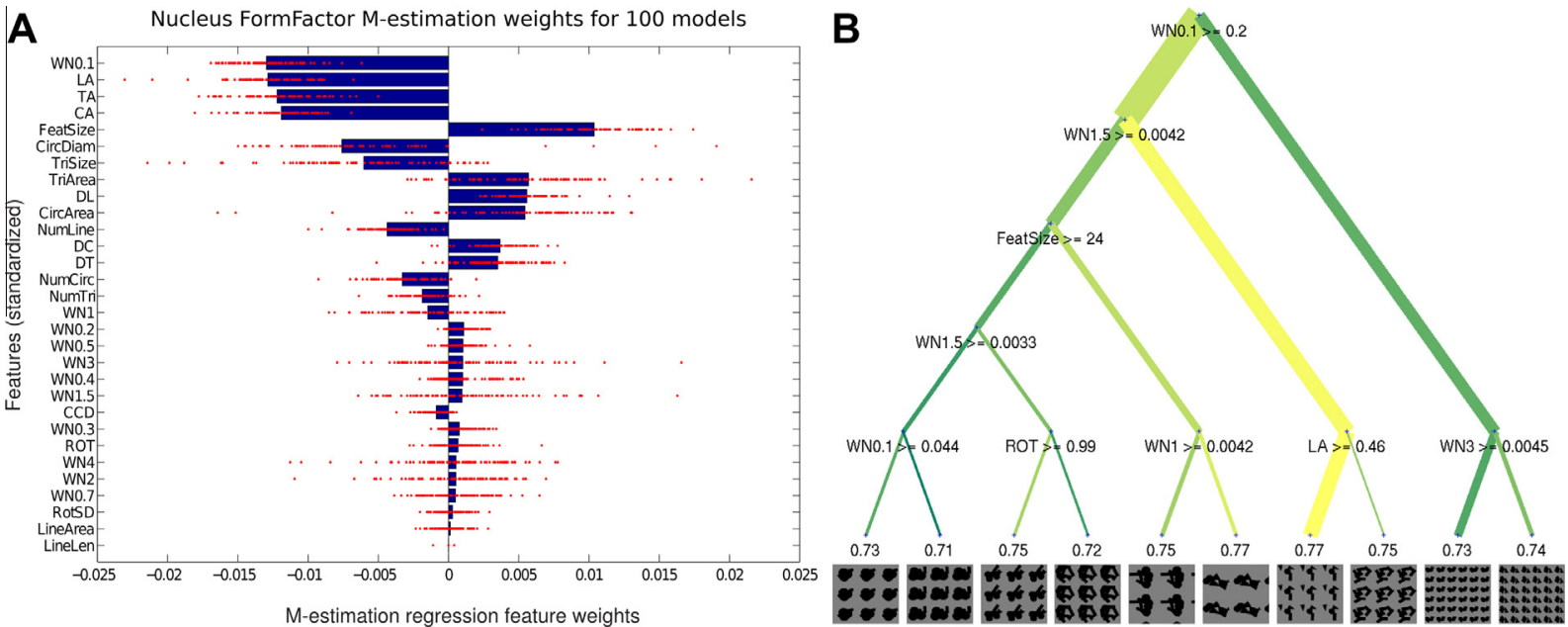

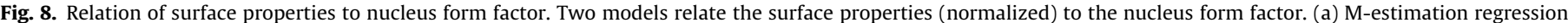

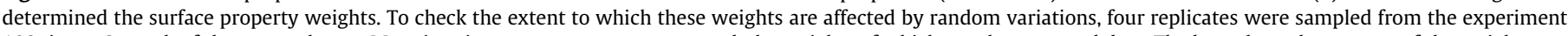

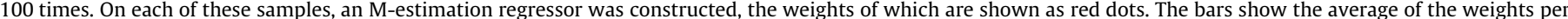

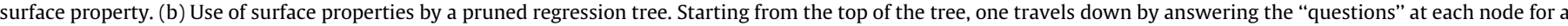

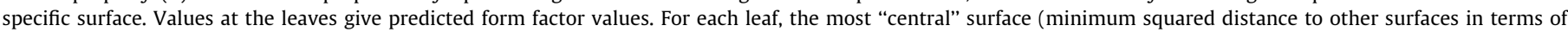

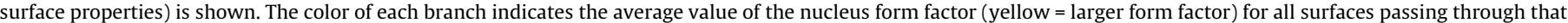
branch, while the thickness of the branch represents the number of surfaces passing through it.

reflects the maximal (2-D) compaction of the nucleus at a certain point (as the nucleus integrated intensity has shown to be relatively unaffected by the surface choice). In Fig. 6 , it is shown that the nuclei with the highest compaction fill the space between four surface features, thereby behaving differently from the cells they belong to, which are guided through the tight grooves between the topographical features.

\subsubsection{Using surface patterns to guide cells}

Contact guidance is a basic behavior of cell alignment on anisotropic surfaces. While cells orientate on a stripe-micropatterned surface without significant surface roughness [31], surface topography can also guide cell orientation. To determine the extent to which the topographies can influence the growth direction of cells, we studied the alignment of the orientation of cells to each other. To that end, we calculated the degree to which cells were oriented in the same direction (see Section 2.1 in Appendix B). Similar to the other features, we ranked the surfaces based on their cell orientation alignment score (Fig. 7). Parallel microgrooves with only minimal or no openings between the grooves result in the best cell orientation alignment. This holds for a range of groove widths. The best cell orientation alignment is achieved with groove widths of $2.5-14.1 \mu \mathrm{m}$ (for the 10 surfaces with the highest cell alignment score). Protrusions of surface primitives into these grooves can cause bottlenecks. Without these protrusions, groove widths range from 5.1 to $16.6 \mu \mathrm{m}$. On the other hand, the width of the openings that connect the main grooves (which are too small for cells) ranges from 0.0 to $4.5 \mu \mathrm{m}$ without protrusions (with $9 / 10$ smaller than $2.6 \mu \mathrm{m}$ ). Of the 10 surfaces with the lowest cell orientation alignment, five induce cells to grow in a crosswise pattern. For these, we observe a minimum groove width of $1.9 \mu \mathrm{m}$ with protrusions and $4.5 \mu \mathrm{m}$ without protrusions.

\subsection{Finding relevant surface properties relevant to promote cell response}

To understand why a particular topography promotes certain cell responses, it is not sufficient to score and rank surfaces. Instead, one needs to understand which property of the topography is beneficial. As the model-based method predicts cell responses on the basis of the topographical properties, learning their weights gives an indication of their relevance. Fig. 8a shows the weights of the (normalized) surface properties when using the M-estimation regression to predict the nucleus form factor based on all 16 replicates. To get an impression of the certainty of the estimated weights, we calculated 100 different M-estimation regressors, each based on four replicates randomly sampled from the 16 replicates available. The red dots indicate the weights of the different models, and show that there is considerable agreement. Hence, the high reproducibility gives confidence to interpret the weights learned.

\subsubsection{Open spaces promote nucleus roundness}

For the nucleus form factor, we observe that the WN0.1 surface property has the highest absolute weight (Fig. 8a). This property captures surface patterns with a small wavelength (a grid-like 
pattern). These high-frequency patterns negatively affect the nuclear form factor, which is related to the roundness of a nucleus (negative weights in Fig. 8a). The LA, TA and CA properties (see Table 1 for definitions) also have high negative weights, indicating that the fractions of surface area covered by lines, triangles and circles, respectively, also affect the nucleus form factor negatively. On the other hand, a large feature size has a positive influence. All these surface properties indicate that large open spaces promote round nuclei. Note that the model makes almost no distinction between circles and triangles, which suggests that the sharpness of the surface features does not play a significant role.

\subsubsection{Finding relevant combinations of surface properties}

Looking at the individual weights of the M-estimation regression does not give any insight into the possible combinatorial interactions between the surface properties necessary to promote a cell response. To determine if such combinatorial interactions could affect our interpretation, we trained a regression tree on all replicate data. The pruning step of the regression tree has a built-in feature selection mechanism. Consequently, the learned tree represents the combinatorial decisions that successfully predict cell responses. Fig. 8b shows the learned regression tree when predicting the nucleus form factor. The tree structure is relatively easy to interpret, and it shows that the combination of a low score for small wavelengths (WN0.1) and a high score for large wavelengths (WN1.5) is enough to separate high scoring surfaces from low scoring ones. The large wavelength property selects for surfaces that have open spaces in a way similar to the combination of LA, TA and CA mentioned in the previous section. Indeed, WN1.5 is negatively correlated with the mean of LA, TA and CA (a Spearman correlation of -0.62). Other decision points in the regression tree are also in good agreement with the individual M-estimation regressions, indicating that there are no noteworthy combinatorial interactions. The regression tree thus confirms that the presence of large open spaces is the most important factor for obtaining round nuclei.

\subsection{Predicting surface responses in silico}

Although our TopoChip tested 2176 surfaces, this is still only a very limited set of possible surface topographies. To explore untested surface topographies we are interested whether we can predict the performance of untested surface topographies for specific cell responses. For that, we can use the regression models that we learned on the basis of the 2176 surfaces. These models predict cell responses and can do so even for unobserved topographies, since one only needs a description of the surface properties, which we can calculate beforehand. To determine if a trained regression model can predict the performance of unseen surfaces accurately, we performed a cross-validation evaluation by repeatedly using a subset of surface topographies for training the regression models and using the remaining surface topographies for testing, i.e. considering them to be unobserved (thus mimicking the situation as if they were measured with a new TopoChip). For the surface topographies used for testing, we can compare the predicted cell responses with the actual measured ones, thus giving an indication of the performance on unseen surfaces. Fig. 9 shows the results for the nucleus form factor prediction using M-estimation regression, which indicates that the predictor accurately predicts the cell responses to new surfaces, with a Pearson correlation of 0.71 between the predicted and actual measurements. Note that we validate the predictions using the cell responses measured for individual surfaces, which, given the limited testing area available per surface, are affected by measurement variability. Therefore, a perfect score cannot be expected. For the Nucleus Maximum Intensity, we obtain a similar score of 0.68 . In contrast, Cell Alignment is

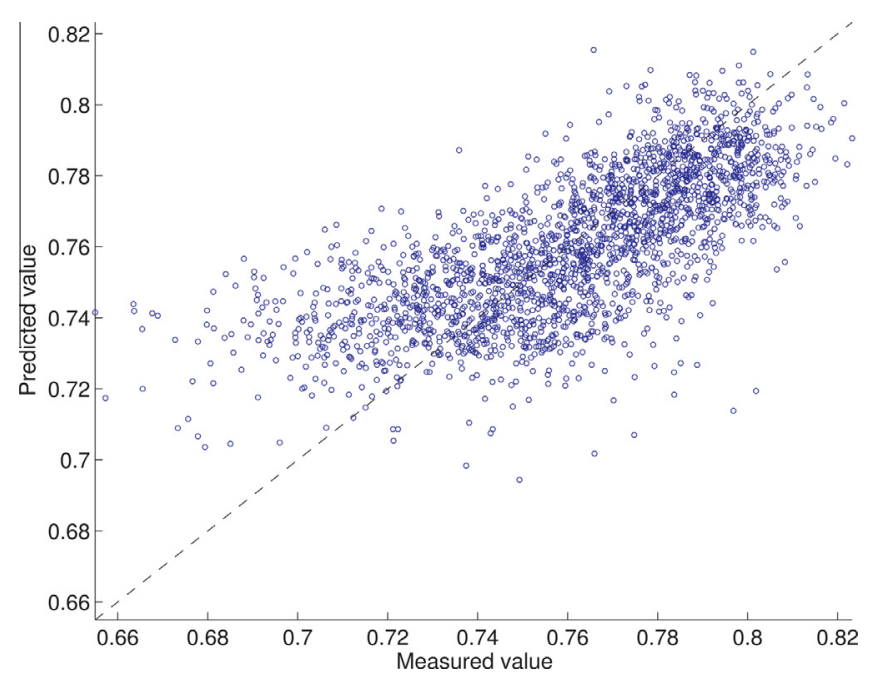

Fig. 9. Performance of the nucleus form factor prediction. Cell surface response predictions vs. observed results for the nucleus form factor.

predicted moderately by the M-estimation regression, attaining a Pearson correlation of 0.25 (this does not improve when using the SVR-RBF approach). This might indicate a limitation in describing the surface topographies using surface properties when predicting Cell Alignment - or that Cell Alignment shows a less consistent surface effect, as observed with the Kruskal-Wallis test. Nevertheless, for most cellular properties we can accurately predict the response for unseen surface topographies (Fig. B.9 in Appendix B).

\section{Discussion}

The advent of high-throughput screening of biomaterial surfaces opens up a completely new view on optimizing surfaces to influence cell behavior. We have presented a number of different computational analyses (surface hit detection and ordering, finding relevant surface properties and predicting cell responses) that illustrate these opportunities. Surface ordering allows us to obtain a (visual) impression of the spectrum of surfaces that induce a certain range of cell behavior. The model-based scoring methods also make it possible to find the particular surface properties that are advantageous for a cell response. This gives insights that open the road towards the rational design of biomaterials. Finally, we showed that the model-based scoring methods are able to predict cell responses for new (unseen) topographies, which could guide both the rational design and experimental optimization of surfaces.

\subsection{Computational implications}

We found that one of the most important criteria in performing the analysis is the statistical robustness of the approaches used. Working with living cells in a miniaturized environment implies a large amount of natural and technical variation. This often causes outliers, to which regular statistics are sensitive. We have shown that proper pre-processing (automatic removal of outliers; see Appendix B) and the use of robust statistics are necessary and promising tools guiding the interpretation of the large amount of data.

Another important observation is that the use of parameterized surfaces in the design of the chip allows one to use machine learning to significantly increase statistical power. This approach, which exploits the similarity of materials, can be seen as an intermediate between testing material gradient surfaces (e.g. $[11,13,14,32])$ and testing discrete surfaces, combining the best of both worlds. 
Similar to gradient surfaces, a large range of possible surfaces can be tested in a single experiment, thereby reducing the chance of methodological errors. Also, results between (slightly) different surfaces can be shared, allowing for an increase in statistical power. On the other hand, similar to the use of discrete surfaces, there is freedom to vary any surface parameter in ways that could not be accomplished using gradients. Also, cells can be grown in confinement on each specific surface, thereby preventing unwanted interactions between cells growing on different surfaces and enabling us to easily determine the corresponding surface parameters for each cell on the chip.

Models based on machine-learning techniques play a large role in this work. They capture cell-surface relations, give insight into what kinds of topography affect cell morphology/behavior and, most importantly, can be extrapolated to new (untested) topographies. This is a relevant step: it is unlikely that one will find the optimal surface through a simple sampling approach. While we were able to validate the predictor through cross-validation techniques, experiments in which one would use such a predictor to suggest more optimal surfaces will have to adopt a combination of high-throughput and iterative testing approaches, in which the results of earlier iterations of surface testing will inform what surfaces to test in subsequent iterations. Only such an active learning approach [33] can truly solve the surface optimization question.

There are also a number of limitations. Explaining cell behavior in modeling terms requires an adequate characterization of the surface designs. For example, our limited ability to model cell alignment is likely due to this issue: by mostly characterizing the surface primitives and not the grooves that separate them, the modeling approach lacks adequate input. Secondly, modeling is not advantageous for cases in which only one of the surfaces shows the interesting cell behavior. In such situations, information cannot be shared across surfaces. However, the large number of (relatively similar) surfaces sampled by the TopoChip makes this situation unlikely to occur.

\subsection{Biological implications}

The results make it clear that surface topographies strongly affect cell morphology, in a reproducible and predictable manner. We found that the statistical models, even given the uncertain measurements, were still highly reproducible (the median rank correlation between independent replicate sets across all morphological properties was 0.77 and 0.88 for the M-estimation regression trained on one and two chips, respectively, and the corresponding values for SVR-RBF were 0.81 and 0.90 ).

We focused here on the relation between surface topography and cell morphology. The control of cell morphology has important consequences for cell behavior and is initiated by cell mechanosensory signals, which, through mechanotransduction systems, affect the activity of the mechanoresponse pathways [34]. While generally it is proposed that these signals are propagated to the nucleus through the use of chemical signals, it has been suggested that the shape of the nucleus in itself also affects cell regulation [35-37]. Recently, it was shown that the dynamic attachment of the genome to the nuclear lamina (which provides mechanical support to the nucleus [38]) correlates with gene expression activity [39]. The spectrum of surfaces as discussed in our work induces a large set of nuclear morphologies (round to elongated, compact to spread out), which can facilitate further study of this connection between nucleus shape and cell regulation. We observed that the shape and orientation of the nucleus is correlated to the cell's shape and orientation. This effect is most likely mediated through the cytoskeleton [36], implying a role for the cytoskeleton as a mechanotransductory system [40]. Consequently, nucleus morphology measurements can be used as a proxy for cell morphology measurements (which can be difficult to measure, due to e.g. cell overlap). Note that this conclusion is justified only for the "ordinary" cases. We observe that the correlation between cell morphology and nucleus morphology breaks down when the nuclei are directly affected by the surface design due to tight space constraints for the cells. This is supported by recent findings in which serious deformation of cell nuclei has been found on micropillar arrays with appropriate parameters, resulting in some very interesting cell nuclei shapes, such as squares, crosses, dumbbells and asymmetrically protruded spheres [41]. Enriching these morphological descriptions with either 3-D or dynamic (over time) measurements might be an interesting avenue.

As well as cell shape, we also looked at cell guidance, which was shown to have a number of important potential applications, e.g. muscle [42] and blood vessel regeneration [43]. Cell guidance through surface topographies has been researched using groove topographies (e.g. [44]). Our investigation of a spectrum of surface patterns confirms the role of microgrooves between features as they rank highest considering how well the orientation of cells are aligned. Additionally, we show the extent to which obstacles may protrude into the microgroove channels. Protrusions (from the sides of the grooves) that result in the smallest width of the groove being approximately $2 \mu \mathrm{m}$ still allow hMSCs to grow through the groove. Non-protruded parts of the groove should, however, measure a minimum of $4.5 \mu \mathrm{m}$ in width, as smaller channels were observed to block cell growth.

The combined use of grooves and protrusions can provide additional control of the cell. In Fig. 6, it can be seen that the combination of a groove and protrusions leads to strong nuclei compaction. In the future, it will be interesting to determine if a combination of grooves and protrusions will allow us to modulate cell behavior beyond just cell guidance.

\subsection{Conclusion}

The statistical and modeling methods discussed in this work enable us to determine surface-affected cellular properties, find spectra of surfaces that modulate such properties, determine the relevant underlying surface parameters and predict cell responses for new surfaces. In particular, the proposed approaches are designed to analyze cell responses to all available surfaces simultaneously, thereby improving statistical power. This approach can be easily applied to study other types of cell responses (e.g. gene activity), or to test the effect of other factors, such as growth factors, gene knockouts or the use of different cell types [45], on cell surface responses. In this way, the methods discussed here could help drive forward the search for optimal biomaterials and related cellular mechanisms.

\section{Disclosures}

J.d.B., B.P. and C.v.B. are shareholders of Materiomics B.V., which commercializes the TopoChip Platform.

\section{Acknowledgements}

M.H. acknowledges the financial support of the STW program. F.H. acknowledges the financial support of the NanoNext NL program, project 06C.12: High throughput screening of biologically active surface nano-topographies.

\section{Appendix A. Figures with essential color discrimination}

Certain figures in this article, particularly Figs. 2-8 are difficult to interpret in black and white. The full color images can be found 
in the on-line version, at http://dx.doi.org/10.1016/j.actbio.2014. 12.019 .

\section{Appendix B. Supplementary data}

Supplementary data associated with this article can be found, in the online version, at http://dx.doi.org/10.1016/j.actbio.2014.12. 019.

\section{References}

[1] Mishra K, Ganju L, Sairam M, Banerjee P, Sawhney R. A review of high throughput technology for the screening of natural products. Biomed Pharmacother 2008;62(2):94-8.

[2] Meyvantsson I, Beebe DJ. Cell culture models in microfluidic systems. Annu Rev Anal Chem 2008;1:423-49.

[3] Unadkat HV, Hulsman M, Cornelissen K, Papenburg BJ, Truckenmüller RK, et al. An algorithm-based topographical biomaterials library to instruct cell fate. Proc Natl Acad Sci 2011;108(40):16565-70.

[4] Kolind K, Leong KW, Besenbacher F, Foss M. Guidance of stem cell fate on 2D patterned surfaces. Biomaterials 2012;33(28):6626-33.

[5] Chen CS, Mrksich M, Huang S, Whitesides GM, Ingber DE. Geometric control of cell life and death. Science 1997;276(5317):1425-8.

[6] Guilak F, Cohen DM, Estes BT, Gimble JM, Liedtke W, et al. Control of stem cell fate by physical interactions with the extracellular matrix. Cell Stem Cell 2009;5(1):17-26.

[7] Yao X, Peng R, Ding J. Effects of aspect ratios of stem cells on lineage commitments with and without induction media. Biomaterials 2013;34(4):930-9.

[8] McBeath R, Pirone DM, Nelson CM, Bhadriraju K, Chen CS. Cell shape, cytoskeletal tension, and RhoA regulate stem cell lineage commitment. Dev Cell 2004;6(4):483-95.

[9] Peng R, Yao X, Ding J. Effect of cell anisotropy on differentiation of stem cells on micropatterned surfaces through the controlled single cell adhesion. Biomaterials 2011;32(32):8048-57.

[10] Pan Z, Qu ZH, Zhang Z, Peng R, Yan C, Ding J. Particle-collision and porogenleaching technique to fabricate polymeric porous scaffolds with microscale roughness of interior surfaces. Chn J Polym Sci 2013;31(5):737-47.

[11] Khung YL, Barritt G, Voelcker NH. Using continuous porous silicon gradients to study the influence of surface topography on the behaviour of neuroblastoma cells. Exp Cell Res 2008;314(4):789-800.

[12] Qu Z, Ding J. Physical modification of the interior surfaces of PLGA porous scaffolds using sugar fibers as template. J Biomater Sci Polym Ed 2013;24(4):447-59.

[13] Veith M, Dufloux C, Ghaemi SR, Aktas C, Voelcker NH. Gradients of $\mathrm{Al} / \mathrm{Al}_{2} \mathrm{O}_{3}$ nanostructures for screening mesenchymal stem cell proliferation and differentiation. Open J Regen Med 2013;2(3):74-9.

[14] Wang PY, Clements LR, Thissen H, Jane A, Tsai WB, Voelcker NH. Screening mesenchymal stem cell attachment and differentiation on porous silicon gradients. Adv Funct Mater 2012;22(16):3414-23.

[15] Zouani OF, Chanseau C, Brouillaud B, Bareille R, Deliane F, Foulc MP, et al. Altered nanofeature size dictates stem cell. J Cell Sci 2012;125:1217-24.

[16] Wang JHC, Jia F, Gilbert TW, Woo SLY. Cell orientation determines the alignment of cell-produced collagenous matrix. J Biomech 2003;36(1):97-102.

[17] Pattison MA, Wurster S, Webster TJ, Haberstroh KM. Three-dimensional, nanostructured PLGA scaffolds for bladder tissue replacement applications. Biomaterials 2005;26(15):2491-500.

[18] Clements LR, Wang PY, Tsai WB, Thissen H, Voelcker NH. Electrochemistryenabled fabrication of orthogonal nanotopography and surface chemistry gradients for high-throughput screening. Lab Chip 2012;12(8):1480-6.
[19] Amis EJ. Combinatorial materials science: reaching beyond discovery. Nat Mater 2004;3(2):83-5.

[20] Meredith JC. Advances in combinatorial and high-throughput screening of biofunctional polymers for gene delivery, tissue engineering and anti-fouling coatings. J Mater Chem 2009;19(1):34-45.

[21] Cranford S, Buehler MJ. Materiomics: biological protein materials, from nano to macro. Nanotech Sci Appl 2010;3:127-48.

[22] Epa V, Burden F, Winkler D, et al. Quantitative structure-property relationship modeling of diverse materials properties. Chem Rev 2012;112(5): 2889-919.

[23] Smith JR, Kholodovych V, Knight D, Kohn J, Welsh WJ. Predicting fibrinogen adsorption to polymeric surfaces in silico: a combined method approach. Polymer 2005;46(12):4296-306.

[24] Li X, Petersen L, Broderick S, Narasimhan B, Rajan K. Identifying factors controlling protein release from combinatorial biomaterial libraries via hybrid data mining methods. ACS Comb Sci 2010;13(1):50-8.

[25] Carpenter AE, Jones TR, Lamprecht MR, Clarke C, Kang IH, et al. Cell Profiler: image analysis software for identifying and quantifying cell phenotypes. Genome Biol 2006;7(10):R100.

[26] Tibshirani R. Regression shrinkage and selection via the lasso. J Roy Stat Soc B 1996:267-88.

[27] Huber PJ, Wiley J. Robust statistics. New York: John Wiley \& Sons; 1981.

[28] Drucker H, Burges CJC, Kaufman L, Smola A, Vapnik V. Support vector regression machines. Adv Neural Inform Process Syst 1997;9:155-61.

[29] Kruskal WH, Wallis WA. Use of ranks in one-criterion variance analysis. J Am Stat Assoc 1952;47(260):583-621.

[30] Dunn OJ. Multiple comparisons among means. J Am Stat Assoc 1961;56(293):52-64.

[31] Sun JG, Tang J, Ding J. Cell orientation on a stripe-micropatterned surface. Chin Sci Bull 2009;54(18):3154-9.

[32] Ruardy T, Schakenraad J, Van der Mei H, Busscher H. Preparation and characterization of chemical gradient surfaces and their application for the study of cellular interaction phenomena. Surf Sci Rep 1997;29(1):3-30.

[33] Settles B. Active Learning Literature Survey. Computer Sciences Technical Report 1648, University of Wisconsin - Madison, 2009

[34] Vogel V, Sheetz M. Local force and geometry sensing regulate cell functions. Nat Rev Mol Cell Biol 2006;7(4):265-75.

[35] Bidwell JP, Alvarez M, Feister H, Onyia J, Hock J. Nuclear matrix proteins and osteoblast gene expression. J Bone Miner Res 1998;13(2):155-67.

[36] Maniotis AJ, Chen CS, Ingber DE. Demonstration of mechanical connections between integrins, cytoskeletal filaments, and nucleoplasm that stabilize nuclear structure. Proc Natl Acad Sci 1997;94(3):849-54.

[37] Hampoelz B, Lecuit T. Nuclear mechanics in differentiation and development. Curr Opin Cell Biol 2011;23(6):668-75.

[38] Aebi U, Cohn J, Buhle L, Gerace L. The nuclear lamina is a meshwork of intermediate-type filaments. Nature 1986;323:560-4.

[39] Peric-Hupkes D, Meuleman W, Pagie L, Bruggeman SWM, Solovei I, et al. Molecular maps of the reorganization of genome-nuclear lamina interactions during differentiation. Mol Cell 2010;38(4):603-13.

[40] Wang N, Tytell JD, Ingber DE. Mechanotransduction at a distance: mechanically coupling the extracellular matrix with the nucleus. Nat Rev Mol Cell Biol 2009;10(1):75-82.

[41] Pan Z, Yan C, Peng R, Zhao Y, He Y, Ding J. Control of cell nucleus shapes via micropillar patterns. Biomaterials 2012;33(6):1730-5.

[42] Zhao Y, Zeng H, Nam J, Agarwal S. Fabrication of skeletal muscle constructs by topographic activation of cell alignment. Biotechnol Bioeng 2009;102(2): 624-31.

[43] Krupinski K, Chu J, Hashi C, Li S. Anisotropic mechanosensing by mesenchymal stem cells. Proc Natl Acad Sci 2006;103(44):16095-100.

[44] Hoffmann-Kim D, Mitchel JA, Bellamkonda RV. Topography, cell response, and nerve regeneration. Annu Rev Biomed Eng 2010;12:203-31.

[45] Biela SA, Su Y, Spatz JP, Kemkemer R. Different sensitivity of human endothelial cells, smooth muscle cells and fibroblasts to topography in the nano-micro range. Acta Biomater 2009;5(7):2460-6. 